\title{
INTERIORIZAÇÃO DO COMBATE À HANSENÍASE E TUBERCULOSE: EXPERIÊNCIAS EXTENSIONISTAS
}

SOUSA, E. do N.1; COSTA, C. O. da ${ }^{2}$ \& NOGUEIRA, P. S. F. ${ }^{3}$

${ }^{1}$ Interna de Enfermagem pela Universidade Federal do Ceará (UFC), integrante da Liga Acadêmica em Doenças Estigmatizantes (LADES) ORCID: https://orcid.org/0000-0003-0730-5790. E-mail: ericanascimento.ufc@ gmail.com; ${ }^{2}$ Interna de Enfermagem pela Universidade Federal do Ceará (UFC). Integrante da Liga Acadêmica em Doenças Estigmatizantes (LADES). ORCID: https://orcid.org/0000-0002-21836224. E-mail: cristinaenfermagemufc@ gmail.com; ${ }^{3}$ Doutora, Professora do Departamento de Enfermagem da Universidade Federal do Ceará (UFC) e coordenadora da LADES ORCID: https://orcid.org/0000-0003-4053-1722. E-mail: sachanogueiraufc@gmail.com.

Artigo submetido em junho de 2020 - DOI 10.32356/exta.v21.n1.43619

\section{RESUMO}

Objetiva-se descrever ações de prevenção e combate à hanseníase e tuberculose, realizadas em município do interior do estado do Ceará, por integrantes da Liga Acadêmica em Doenças Estigmatizantes (LADES) enquanto internos de Enfermagem no programa Centro Rural Universitário de Treinamento e Ação Comunitária (CRUTAC) do curso de Enfermagem da Universidade Federal do Ceará (UFC). Trata-se de um relato de uma série de experiências extensionistas desenvolvidas em uma Unidade Básica de Saúde (UBS), no município de Umirim, Ceará, nos dias 24 e 29 de março, e 24 de maio de 2019. Realizou-se, educação em saúde por meio de estratégias como roda de conversa e sala de espera, capacitação de Agentes Comunitários de aúde (ACS) e divulgação em rádio local sobre sinais e sintomas da hanseníase com chamada da comunidade para o dia " $\mathrm{D}$ " de avaliação e prevenção de incapacidades por hanseníase com avaliação neurológica simplificada. Conclui-se que a interiorização de ações de saúde como as aqui relatadas permitem descentralizar e socializar o conhecimento científico desenvolvido na universidade e proporcionam a vivência de conceitos e fundamentos teóricos aprendidos por estudantes, permitindo consolidar e expandir conhecimentos, ao passo que sensibiliza a população frente a problemas de saúde pública importantes que assolam comunidades interioranas.

PALAVRAS-CHAVE: Hanseníase. Tuberculose. Educação em saúde.

\section{INTERIORIZATION OF THE FIGHT AGAINST LEPROSY AND TUBERCULOSIS: EXTENSIONIST EXPERIENCES}

\begin{abstract}
The objective is to describe actions to prevent and combat leprosy and tuberculosis, carried out in a city in the interior of the state of Ceará by members of the Academic League on Stigmatizing Diseases (LADES) as nursing interns in the Rural University Training and Community Action Program (CRUTAC) of the Nursing course at the Federal University of Ceará (UFC). It is a report of a series of extension experiences developed in a Basic Health Unit (UBS), in the municipality of Umirim, Ceará, on March 24th and 29th, and May 24th, 2019. Education took place in health through strategies such as a conversation circle and a waiting room, training of Community Health Agents (CHA) and
\end{abstract}

issemination on local radio about signs and symptoms of leprosy with a call from the community for "D" day of evaluation and prevention of leprosy disabilities with simplified neurological assessment. It is concluded that the internalization of health actions such as those reported here allows to decentralize and socialize the scientific knowledge developed at the university center and provide the experience of concepts and theoretical foundations learned by students, allowing the consolidation and expansion of knowledge, while it sensitizes the population to important public health problems plaguing rural communities. 


\section{INTRODUÇÃO}

A hanseníase e a tuberculose (TB) estão incluídas no grupo de doenças negligenciadas pela Organização Mundial de Saúde (OMS), e afetam a vida de milhões de pessoas em todo o mundo. As populações mais afetadas são as que vivem em situações de pobreza, em moradias precárias, inseguras, e que dispõem de acesso limitado às ações e serviços de saúde (SANTOS, 2019).

O Brasil definiu, em 2006, como doenças negligenciadas aquelas que além de prevalecerem em condições de pobreza, também contribuem para a manutenção do quadro de desigualdades no país, representando forte entrave ao desenvolvimento. Cabe ressaltar, que quanto melhores as condições de saúde de um país, melhores serão seus índices de desenvolvimento econômico e social entre as nações (OLIVEIRA, 2018).

O risco de contaminação por alguma doença negligenciada, na população mundial, é de $40 \%$, ainda assim, elas permanecem ocultas, não diagnosticadas, concentradas em áreas rurais, ou em favelas urbanas, locais menos privilegiados (ANDRADE, 2015).

A hanseníase e a TB vêm se destacando como problema de saúde pública no Brasil há anos devido a sua magnitude, transcendência e vulnerabilidade. Atualmente, o país é o segundo lugar mundial em casos de hanseníase, atrás apenas da Índia, e está na vigésima posição entre países com maior carga de TB no mundo (SILVA, 2017; MARQUES, 2019).

Ambas são doenças de alta infectividade, transmitidas através da tosse, fala ou espirro, de pessoa a pessoa, e dependem de fatores imunológicos para sua progressão, mas há certas peculiaridades entre elas. O agente etiológico da TB, Mycobacterium tuberculosis, permanece em dispersão no ar por mais tempo, devido ao seu menor tamanho, e por ser veiculado em aerossóis, enquanto a hanseníase carece de contato prolongado e carga elevada do agente, Mycobacterium Leprae, para sua evolução (BRASIL, 2017b, 2017c; BRASIL, 2019).

$\mathrm{Na}$ hanseníase, uma das principais preocupações é com seu alto potencial de gerar incapacidades, quando não diagnosticada em tempo oportuno, fato causador de estigma social e perda da qualidade de vida. Já na tuberculose, o que mais preocupa é a coinfecção TB e Vírus da Imunodeficiência Humana (HIV).

Estratégias educativas que sensibilizem a população na busca ativa de sintomáticos respiratórios de TB e pessoas com sintomas sugestivos de hanseníase como lesões na pele, alteração de sensibilidade, ou alterações neurais, podem contribuir para o diagnóstico precoce e tratamento oportuno, podendo reduzir as taxas de abandono de tratamento e de transmissão, 
consequentemente as morte decorrentes da doença (SACKSER, 2019; TAVARES, 2019).

Por conseguinte, ao reconhecer o risco acrescido dessas doenças na sociedade, destacase a relevância das ações aqui descritas, à medida que incentivam mais ações de prevenção e combate às doenças negligenciadas, promovem saúde em regiões com menos facilidade de acesso, fortalecem o vínculo entre ensino e serviços de saúde, e reforçam que as ações de vigilância devem ser priorizadas, de forma contínua e sistemática.

Assim, o objetivo principal deste estudo é descrever as ações de prevenção e combate à hanseníase e tuberculose, desenvolvidas no interior do estado do Ceará, por estudantes, internos de Enfermagem no programa Centro Rural Universitário de Treinamento e Ação Comunitária (CRUTAC).

\section{MATERIAIS E MÉTODOS}

Trata-se de um estudo descritivo do tipo relato de experiência, acerca de ações extensionistas de prevenção e combate à hanseníase e à tuberculose, desenvolvidas por integrantes da Liga Acadêmica em Doenças Estigmatizantes (LADES) enquanto internos de Enfermagem no Centro Rural Universitário de Treinamento e Ação Comunitária (CRUTAC), programa da Universidade Federal do Ceará (UFC) para alunos do último ano dos cursos da área da saúde.

As ações relatadas neste estudo foram desenvolvidas nos dias 24 e 29 de março de 2019, pela celebração do dia mundial de combate à tuberculose, e no dia 24 de maio, do mesmo ano, por ocasião do dia estadual de combate à hanseníase no Ceará. Essas são datas alusivas estratégicas, nas quais há maior mobilização dos profissionais de saúde da atenção básica para busca ativa e detecção precoce de novos casos destas comorbidades.

O CRUTAC teve início na UFC em 1972 com a Resolução nº 259/72 e, atualmente, o é estágio curricular obrigatório para os cursos de Medicina, Enfermagem e Odontologia. Entre os principais objetivos do programa destacam-se: motivar a produção de conhecimentos e pesquisas dentro da realidade de municípios de pequeno e médio porte; permitir experiências de educação em saúde em contextos culturais diversos; possibilitar conhecimento e habilidades para resolver ou bem encaminhar os problemas de saúde mais prevalentes em população de município do interior (UNIVERSIDADE FEDERAL DO CEARÁ, 2021).

O município, cenário das ações realizadas, foi Umirim-CE, que se encontra a $100 \mathrm{~km}$ de Fortaleza, capital do estado. De clima semiárido, área territorial de 316.724 km², e população 
estimada para 2018 de 19.811 habitantes (IBGE, 2019; UMIRIM, 2019).

A UBS que recebeu os estudantes é uma das sete que abrangem o território, e fica localizada em sua sede. Esta funciona semanalmente, de segunda à sexta-feira, e cobre uma população de cerca de três mil e quinhentas pessoas (UMIRIM,2019).

As ações de promoção da saúde, prevenção e combate à hanseníase e TB, descritas em resultados e discussões e apresentadas no quadro 1, foram planejadas a partir da iniciativa dos estudantes, tendo como embasamento teórico-científico as orientações dos manuais do ministério da saúde (BRASIL, 2017c, 2019), e o apoio da equipe da UBS e da secretaria de saúde do município.

Quadro 1- Síntese das atividades desenvolvidas no CRUTAC nos meses de março e maio para prevenção e combate à hanseníase e TB. Umirim, Ceará, Brasil 2019.

\begin{tabular}{|c|c|c|c|}
\hline $\begin{array}{l}\text { Mês de } \\
\text { execução }\end{array}$ & Atividade(s) realizada(a) & Objetivo(s) & Público alcançado \\
\hline \multirow{2}{*}{ Março } & $\begin{array}{l}\text { Educação em saúde sobre } \\
\text { tuberculose com a } \\
\text { população local }\end{array}$ & $\begin{array}{l}\text { Sensibilizar a população para } \\
\text { o combate à TB com o foco } \\
\text { na prevenção e promoção da } \\
\text { saúde }\end{array}$ & - Cerca de vinte pessoas \\
\hline & $\begin{array}{l}\text { Capacitação dos Agentes } \\
\text { de Saúde do município } \\
\text { sobre Tuberculose }\end{array}$ & $\begin{array}{l}\text { - Capacitar os ACSs sobre os } \\
\text { aspectos gerais da tuberculose } \\
\text { com foco no diagnóstico } \\
\text { precoce e tratamento efetivo }\end{array}$ & $\begin{array}{l}\text { - Vinte e cinco Agentes } \\
\text { Comunitários de Saúde de um } \\
\text { total de trinta e cinco existentes } \\
\text { no município. }\end{array}$ \\
\hline \multirow[t]{2}{*}{ Maio } & $\begin{array}{l}\text { Realização do dia “D” de } \\
\text { prevenção de } \\
\text { incapacidades e busca de } \\
\text { casos suspeitos de } \\
\text { hanseníase }\end{array}$ & $\begin{array}{l}\text { - Divulgar em rádio dia e local } \\
\text { de realização do evento, com } \\
\text { foco na busca ativa de } \\
\text { manchas suspeitas }\end{array}$ & $\begin{array}{l}\text { - Toda a comunidade do } \\
\text { município que teve acesso ao } \\
\text { programa no qual foi divulgado }\end{array}$ \\
\hline & $\begin{array}{l}\text { Sala de espera sobre } \\
\text { hanseníase }\end{array}$ & $\begin{array}{c}\text { - Realizar busca ativa e } \\
\text { promoção da saúde }\end{array}$ & - Cerca de quinze pessoas \\
\hline
\end{tabular}

Fonte: Autores, 2019.

As estratégias utilizadas para realização das atividades educativas foram a roda de conversa e exposição dialogada para capacitação profissional. 
Na capacitação com os ACS foi utilizado um questionário simples contendo nove perguntas de temas básicos sobre tuberculose, as quais deveriam ser assinaladas com $\mathrm{V}$ para as afirmativas verdadeiras, e F para as falsas. As temáticas propostas na avaliação foram: a forma de transmissão da TB, seu agente etiológico, a não transmissão de TB por objetos pessoais, a não infecção num primeiro contato, a manifestação tardia da doença, sobre quem são os sintomáticos respiratórios, sobre os riscos para desenvolver TB, sobre quem deve fazer a baciloscopia de escarro, sobre a quebra de transmissão após 15 dias do início do tratamento.

A avaliação foi aplicada antes da intervenção educativa para identificar as lacunas de conhecimento do público-alvo, e após a atividade para que os participantes avaliassem sua evolução, como agentes ativos na própria formação continuada.

Este estudo não necessitou da apreciação do Comitê de Ética em Pesquisa, pois não foram utilizados dados relativos a sujeitos ou coletivos, apenas relato das experiências vivenciadas pelos internos.

\section{RESULTADOS E DISCUSSÕES}

No dia 24 de março, realizou-se ação de educação em saúde pela manhã, o público-alvo foram as pessoas que estavam aguardando atendimento na unidade de saúde. Cerca de vinte pessoas foram alcançadas com a atividade. O local da atividade foi a entrada da UBS, a qual foi decorada de forma a celebrar o dia mundial de combate à tuberculose, com cartazes, balões e enfeites, foi disponibilizado café da manhã gratuito aos participantes. Como tecnologia educativa usou-se a roda de conversa sobre aspectos gerais da doença como: transmissão, sinais e sintomas, tratamento, possibilidade de cura, mitos e verdades, preconceito e superação. Durante a atividade, também surgiram dúvidas, questionamentos e falas importantes dos populares, o que ajudou a complementar a exposição.

Com foco na promoção da saúde, a educação popular em saúde tem a roda de conversa como uma das principais estratégias educativas e de baixo custo. Este é um método de discussão que possibilita aprofundar o diálogo com a participação democrática, a partir das vivências que cada pessoa possui do assunto, permitindo os participantes expressarem suas impressões, conceitos, opiniões e concepções sobre o tema proposto, assim como trabalhar reflexivamente as manifestações apresentadas pelo grupo (DIAS et al., 2018). 
A atividade de capacitação dos ACS foi realizada em um ambiente separado da UBS, uma sala de reuniões, na qual ocorrem reuniões mensais com a equipe. A exposição dos slides se deu pela smart TV da unidade. Antes do início da ação, foram disponibilizados ao grupo uma folha simples contendo nove questões básicas sobre TB, para avaliar o conhecimento prévio e as principais dúvidas que deveriam ser sanadas durante a capacitação. Após a estratégia educativa os agentes responderam novamente as mesmas questões, para que os mesmos reconhecessem no que melhoraram após a intervenção. As questões com maior índice de erro antes da capacitação foram sobre: a forma de transmissão da TB (52\%); o agente etiológico (78\%); a não infecção no primeiro contato (74\%), a não transmissão por objetos pessoais como talheres, copos, roupas, e outros (70\%). Na avaliação aplicada após a capacitação o número de erros nas mesmas questões ficou abaixo de $20 \%$.

Ressalta-se, que atividades de formação, capacitação e aperfeiçoamento são de suma importância para este público, favorecendo o correto manejo das doenças e agravos. Segundo a Política Nacional de Atenção Básica (PNAB), são funções dos ACS: desenvolver atividades de promoção da saúde, de prevenção de doenças e agravos, em especial aqueles mais prevalentes no território, e de vigilância em saúde, por meio de visitas domiciliares regulares e de ações educativas individuais e coletivas, na UBS, no domicílio e outros espaços da comunidade, incluindo a investigação epidemiológica de casos suspeitos de doenças e agravos junto a outros profissionais da equipe quando necessário (BRASIL, 2017a).

Em 24 de maio, comemora-se no Ceará o dia estadual de controle da hanseníase. Oportunamente, uma semana antes desta data, através da rádio local, buscou-se enfatizar a importância da detecção precoce, para o controle e tratamento da hanseníase, bem como seu potencial de causar incapacidades/deficiências. Uma vinheta explicativa trazia informações sobre a forma de transmissão, e fazia um alerta para manchas brancas, avermelhadas ou acastanhadas na pele, as quais merecem ser avaliadas na unidade de saúde, divulgava ainda que ocorreria um evento nomeado 'Dia "D" de prevenção de incapacidades e busca de casos suspeitos de hanseníase', acessível a toda a comunidade, no qual haveria uma equipe treinada para fazer a avaliação dermatoneurológica e dar seguimento ao tratamento, se identificada a doença.

O dia "D" iniciou-se com a realização de uma atividade de sala de espera para o público que compareceu à unidade, aproximadamente 15 pessoas. Utilizou-se, no primeiro momento, 
do método de exposição de slides e roda de conversa sobre sinais, sintomas e curiosidades sobre a hanseníase, e em seguida, a equipe da LADES ofertou atendimento aos casos suspeitos. A duração foi de um turno. Foram avaliados, em consulta individual, dois casos suspeitos, que foram descartados, e um paciente que estava em tratamento obteve avaliação dermatoneurológica.

Importa salientar, que ações que despertam o interesse da população por diferenciar manchas comuns de manchas por hanseníase, colaboram para a identificação de casos índices de forma precoce. A OMS recomenda a busca ativa como parte do programa de eliminação da hanseníase. Nota-se, que além de sua importância na identificação de novos casos, a busca ativa constitui uma ferramenta útil na profilaxia da doença em áreas de alta prevalência ou para o controle de abandonos e de comunicantes (LIMA et al., 2016).

Como limitação do estudo destaca-se a indisponibilidade de recursos financeiros dos envolvidos para empreender maior número de ações, e de maior abrangência, bem como a não mensuração do impacto social e alcance delas de forma contínua.

\section{CONCLUSÃO}

O CRUTAC apresenta-se como uma via que facilita o alcance da promoção da saúde para a população, à medida que interioriza ações de saúde, levando jovens, ainda em formação, à prática profissional nos serviços de saúde do interior do estado.

Conclui-se portanto, que, com a interiorização de ações extensionistas, é possível descentralizar e socializar o conhecimento científico, levando ao interior das comunidades carentes o que se vivencia na universidade, no centro das grandes cidades, de forma a promover saúde em locais mais remotos.

As ações, com apoio da LADES no CRUTAC, proporcionaram a aplicação prática dos conceitos e fundamentos aprendidos em sala de aula, permitindo consolidar e expandir conhecimentos, ao passo que propõe sensibilizar a população frente ao problema de saúde pública da hanseníase e da tuberculose no estado.

\section{REFERÊNCIAS}

ANDRADE, B.L.A.; ROCHA, D.G. Doenças negligenciadas e bioética: diálogo de um velho problema com uma nova área do conhecimento. Revista Bioética, v. 23, n. 1, p.105-113, abr. 2015. FapUNIFESP (SciELO). Disponível em: <http://dx.doi.org/10.1590/1983- 
80422015231051>. Acesso em: 25 fev. 2020.

BRASIL. Manual de Recomendações para o Controle da Tuberculose no Brasil. Departamento de Vigilância das Doenças Transmissíveis - Brasília: Ministério da Saúde, 2019.

Ministério da Saúde. Gabinete do Ministro. Portaria No 2.436, de 21 de setembro de 2017a. Aprova a Política Nacional de Atenção Básica, estabelecendo a revisão de diretrizes para a organização da atenção básica no âmbito do Sistema Único de Saúde (SUS).

Disponível em:

<http://bvsms.saude.gov.br/bvs/saudelegis/gm/2017/prt2436_22_09_2017.html>. Acesso em: 27 fev. 2020.

. Ministério da Saúde. Secretaria de Vigilância em Saúde. Brasil livre da

tuberculose: Plano nacional pelo fim da tuberculose como problema de saúde pública. 52 p. Brasília (DF), 2017 b.

Guia prático sobre a hanseníase. Secretaria de Vigilância em Saúde. Departamento de Vigilância e Doenças Transmissíveis. Ministério da Saúde: Brasília. 2017c.

Ministério do Planejamento, Orçamento e Gestão. Instituto Brasileiro de Geografia e Estatística. Umirim. Disponível em:

<https://cidades.ibge.gov.br/brasil/ce/umirim/panorama>. Acesso em: 15 de agosto de 2019.

DIAS, E.S.M., et al. Roda de conversa como estratégia de educação em saúde para a enfermagem. Rev Fund Care Online. v. 10, n. 2, p. 379-384. abr/jun, 2018. Disponível em: <http://dx.doi.org/10.9789/2175- 5361.2018.v10i2.379-384> Acesso em: 27 fev. 2020.

LIMA, R.S.K. et al. A importância da busca ativa como estratégia de controle da hanseníase em territórios endêmicos. Hansen Int. v. 41, n. 2, p. 55-63, 2016.

MARQUES, W.S. et al. Características clínicas e epidemiológicas de idosos com hanseníase atendidos em um Hospital de Ensino no Nordeste do Brasil. Enfermagem Brasil, v. 18, n. 3, p.406-413, 16 jul. 2019. Disponível em: <http://dx.doi.org/10.33233/eb.v18i3.2505>. Acesso em: 25 fev. 2020.

OLIVEIRA, R.G. Sentidos das Doenças Negligenciadas na agenda da Saúde Global: o lugar de populações e territórios. Ciência \& Saúde Coletiva, v. 23, n. 7, p.2291-2302, jul. 2018. Disponível em: <http://dx.doi.org/10.1590/1413-81232018237.09042018>. Acesso em: 25 fev. 2020.

SACKSER, M. A. Razões que levam os pacientes com tuberculose a abandonarem o tratamento: perspectivas do enfermeiro. Revista Enferm Atual, v. 87, n. 25, p.01-10, 2019. Disponível em: <http://revistaenfermagematual.com/index.php/revista/article/view/214>. Acesso em: 19 mar. 2020. 
SANTOS, G.R.A. O desequilíbrio Fatal: Reflexão sobre as Doenças Negligenciadas. 2019. 49 f. Monografia (Graduação) - Curso de Relações Internacionais, Departamento de Relações Internacionais, Universidade Federal da Paraíba, João Pessoa, 2019. Disponível em: <https://repositorio.ufpb.br/jspui/bitstream/123456789/15559/1/GRAF05092019.pdf>. Acesso em: 17 jan. 2020.

SILVA, I.G.B. et al. Liga acadêmica de doenças negligenciadas: encurtando laços entre ensino, serviço e gestão. Revista Saúde e Ciência online, v. 6, n. 3, p 22-30, 2017. ISSN:2317-8469. Disponível em:

https://rsc.revistas.ufcg.edu.br/index.php/rsc/article/view/183/179. Acesso em: 15 fev. 2019.

TAVARES, J.B. Método problematizador no trabalho de busca ativa de hanseníase em dois centros de saúde: descrição de experiência. Revista Brasileira de Educação e Saúde, Pombal, v. 9, n. 2, p.01-06, 11 mar. 2019. Disponível em:

<https://editoraverde.org/gvaa.com.br/revista/index.php/REBES/article/view/6192/5586>. Acesso em: 19 mar. 2020.

UMIRIM. Prefeitura de Umirim. Disponível em:

<http://www.umirim.ce.gov.br/omunicipio.php>. Acesso em: 15 de agosto de 2019.

UNIVERSIDADE FEDERAL DO CEARÁ. Departamento de Saúde Comunitária. Disponível em: <http://www.saudecomunitaria.ufc.br/extensao/crutac/>. Acesso em: 15 de agosto de 2021. 